Acidosis

branched chain amino aciduria

butanone

genetic disorder

isoleucine $\alpha$-methylacetoacetate

$\alpha$-methyl- $\beta$-hydroxybutyrate

thiolase

$N$-tiglylglycine

\title{
An Inherited Disorder of Isoleucine Catabolism Causing Accumulation of $\alpha$-Methylacetoacetate and $\alpha$-Methyl- $\beta$-hydroxybutyrate, and Intermittent Metabolic Acidosis
}

\author{
Robert S. Daum, Gharles R. Sariver ${ }^{[32]}$, Orval A. Mamer, Edgard Delvin, \\ Peter lamm, and Hy Goldman \\ Departments of Biology, Pediatrics and Medicine, Faculties of Science and Medicine, McGill University, \\ Montreal, Quebec, Canada
}

\section{Extract}

At least 15 apparently inherited disorders of branched chain amino acid catabolism are now known; the 12th in chronological order of discovery is described in this report. It is a partial defect of the pathway of isoleucine oxidation beyond the level of oxidative decarboxylation and prior to the oxidation of propionate. The impairment of isoleucine catabolism appears to be situated at the "thiolase" reaction which converts $\alpha$-methylacetoacetyl coenzyme A (CoA) to propionyl-CoA and acetyl-CoA.

Two pedigrees ( $\mathrm{B}$ and $\mathrm{M}$ ) were investigated in detail. A third (S pedigree) has been brought to our attention for analysis of metabolites in urine but we have not performed additional studies in the latter. Each propositus was ascertained because of intermittent, odorless metabolic acidosis usually precipitated by intercurrent infection. Lethargy and coma occurred frequently during the periods of acidosis. One M sib, also presumably affected, died abroad in such an episode. Symptoms can be ameliorated by a low protein diet and careful attention to the management of intercurrent illness.

A large excess of $\alpha$-methyl- $\beta$-hydroxybutyrate and a seemingly smaller excess of $\alpha$-methylacetoacetate is present at all times in the urine of the three propositions. The $\mathrm{M}$ and $\mathrm{S}$ propositi also excrete $N$-tiglylglycine. The amounts of these unusual metabolites increase severalfold during acidosis and after a dietary load of L-isoleucine $(75 \mathrm{mg} / \mathrm{kg}, 3$ times daily for 2 days). The urine also contains butanone, particularly during acidosis. The amount of propionate and of glycine and other amino acids in blood and urine is always normal in our patients. Oxidation of L-isoleucine-U-U $-{ }^{14} \mathrm{C}$ to $\mathrm{CO}_{2}$ by cultured skin fibroblasts is about $45 \%$ of normal in the $\mathrm{B}$ propositus. The precise nature and location of the enzyme defect awaits clarification.

Studies of family members reveal that presumed obligate heterozygotes excrete a small excess of $\alpha$-methyl- $\beta$-hydroxybutyrate at all times; the amount can be increased by L-isoleucine feeding. The condition is apparently inherited in autosomal recessive fashion. It is likely that more than one form of mutant allele is responsible for the condition, as it is found in the three different pedigrees described here.

\section{Speculation}

Investigation of "unexplained," intermittent metabolic acidosis in childhood has led to the discovery of a "new" disorder of branched chain amino acid catabolism. Gas 
chromatography coupled with mass spectrometry were important aids to the diagnosis. Rapid escalation of acidosis during catabolic episodes encourages one to suspect that specific metabolites, themselves accumulating during episodic illness, may further inhibit the mutant enzyme. A temperature-sensitive mutant enzyme was not identified in cultured skin fibroblasts.

\section{Introduction}

In recent years, 15 disorders of branched chain amino acid metabolism have been described (Fig. 1). The patients presented in this report have one of these "new" diseases [1]. Isoleucine catabolism is impaired and we believe the evidence indicates a partial block at the thiolase step in which $\alpha$-methylacetoacetyl-CoA $(\alpha$ MAA) is split into its products, acetyl-CoA and propionyl-CoA. The block causes accumulation of $\alpha$-MAA, its immediate precursor $\alpha$-methyl- $\beta$-hydroxybutyric acid $(\alpha-\mathrm{M} \beta \mathrm{HB})$, and the glycine conjugate of another precursor, tiglic acid. As with many other inborn errors of branched chain amino acid catabolism, the presenting clinical feature was severe, recurrent, metabolic acidosis. This report describes the case histories of patients from two separate pedigrees, the evidence for presumed autosomal recessive inheritance of the condition, and the investigations which were carried out to elicit the apparent nature of the biochemical defect.

\section{Case Reports}

\section{$B$ Pedigree}

Patient B,III,2 is the propositus who led us to the discovery of the disease [1]. He was born in 1965 after a normal gestation and delivery. Breast feeding was pursued until 6 months of age and the patient remained well until 22 months when he was first admitted to the Montreal Children's Hospital after 2 days of fever, rhinorrhea, and poor appetite. On the day of admission, there was profuse vomiting and diarrhea,
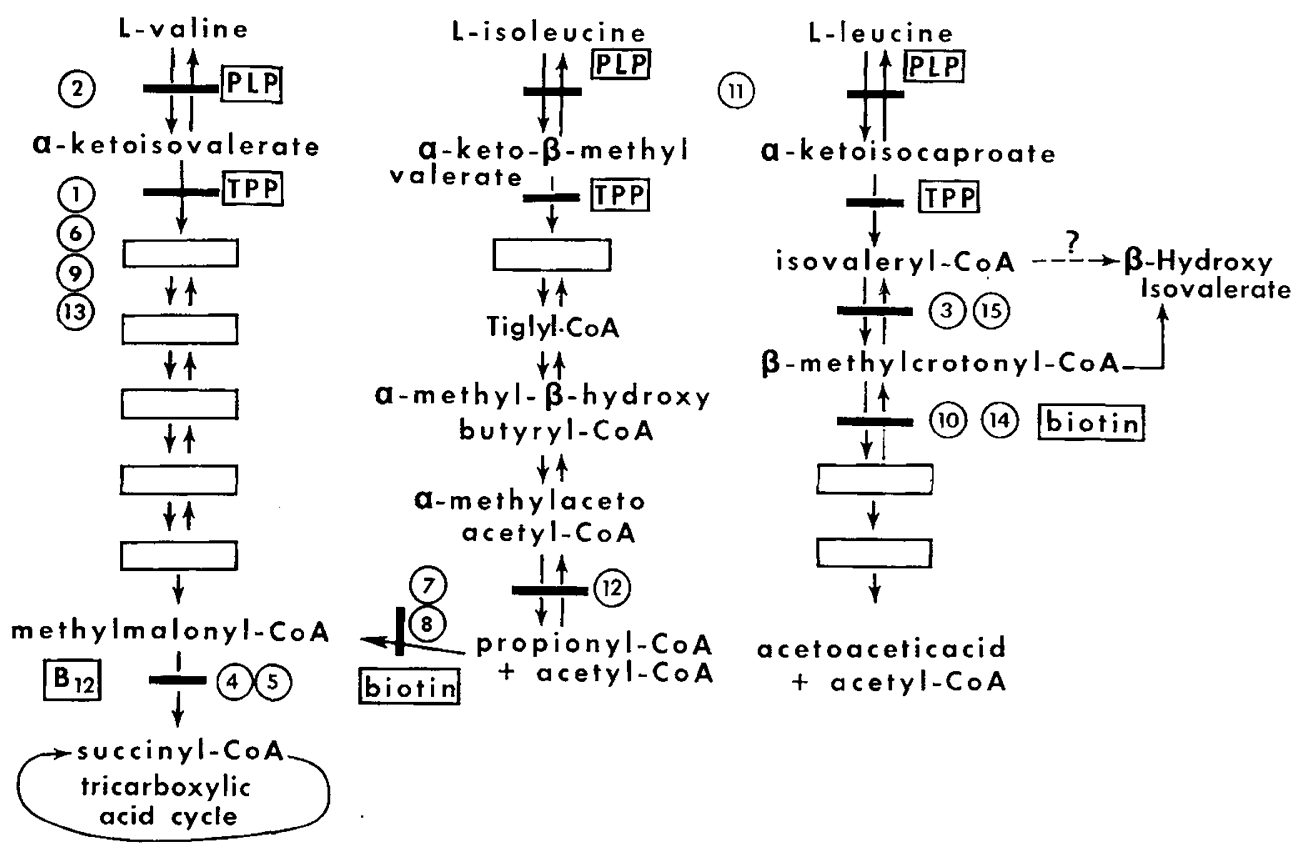

Fig. 1. Metabolism of branched chain amino acids. Known disorders of branched chain amino acid metabolism are shown by a number representing the order in which the respective traits have been reported. The metabolities which accumulate in body fluids in the various conditions are named; other metabolites in the pathways are shown by open boxes. Tiglate and $\beta$-methylcrotonate have been recovered in urine as their glycine conjugates, $N$-tiglylglycine and $\beta$-methylcrotonylglycine, respectively. The presumed direct conjugation reactions are not shown. The reaction presumably affected in the patients reported here is reaction 12. PLP: Pyridoxal phosphate; TPP: thiamine pyrophosphate. 
Table $I$. Blood chemistries in propositus of B pedigree

\begin{tabular}{|c|c|c|c|c|c|c|c|}
\hline Age & $\begin{array}{l}\text { Admission } \\
\text { temperature, } \\
{ }^{\circ} \mathrm{C}\end{array}$ & $\begin{array}{l}\mathrm{PCO}_{\mathrm{C}} \\
\mathrm{mm} \mathrm{Hg}\end{array}$ & $\mathrm{pH}$ & $\underset{\mathrm{mEq} / \mathrm{liter}}{\mathrm{HCO}_{3}}$ & Clinical state & Clinical diagnosis & $\begin{array}{l}\text { "Unknown } \\
\text { compound" } \\
\text { in urine }\end{array}$ \\
\hline 1 yr $5 \mathrm{mo}$ & $38.3^{\circ}$ & 28.2 & 6.99 & 8.0 & Comatose & $\begin{array}{l}\text { Acidosis secondary to poi- } \\
\text { son ingestion }\end{array}$ & $?$ \\
\hline 2 yr $10 \mathrm{mo}$ & 38.9 & 23.1 & 7.33 & 14.8 & Semicomatose & $\begin{array}{l}\text { Acidosis secondary to phe- } \\
\text { nol poisoning }\end{array}$ & ? \\
\hline $4 \mathrm{yr}$ & 38.3 & 19 & 6.95 & 9.0 & Comatose & $\begin{array}{l}\text { Acidosis secondary to ru- } \\
\text { bella }\end{array}$ & ++++ \\
\hline $4 \mathrm{yr} 10 \mathrm{mo}$ & 38.3 & 25 & 7.33 & 19 & Alert & Methylmalonic aciduria & ++++ \\
\hline $4 \mathrm{yr} 10 \mathrm{mo}$ & 38.9 & 31 & 7.36 & 18 & Alert & Methylmalonic aciduria & +++ \\
\hline $5 \mathrm{yr}$ & 37.2 & 40 & 7.44 & 26.4 & Alert & New disease & + \\
\hline
\end{tabular}

and the child had become semicomatose and moderately dehydrated. The liver was $1 \mathrm{~cm}$ below the costal margin. Deep tendon reflexes were brisk and symmetrical but the plantar reflexes were upgoing. The respiratory rate was $38 / \mathrm{min}$ and blood gas studies revealed a profound metabolic acidosis (Table I). Chemical analyses of blood were normal for $\mathrm{Na}, \mathrm{K}, \mathrm{Cl}$, glucose, lactate, blood urea nitrogen, alkaline phosphatase, and serum glutamine oxaloacetic transaminase. An electrocardiogram revealed prolongation of the QT interval. The electroencephalogram revealed a mild, diffuse disturbance. He was treated with intravenous bicarbonate and electrolyte solutions and the clinical picture improved within 2 days. A diagnosis of metabolic acidosis, secondary to accidental ingestion of a poison was entertained because the boy lived on a dairy farm where toxic chemicals were stored.

The child presented again with a similar clinical picture when 34 months old. He had been well until 4 days before, when he had developed an upper respiratory tract infection followed by vomiting, hyperventilation, and irritability. He was semicomatose at the time of admission. The blood pressure was $90 / 68$; pulse $140 / \mathrm{min}$ and regular; temperature, $102^{\circ} \mathrm{F}$; respirations $60 / \mathrm{min}$. An acetone-like odor was noted to the breath and metabolic acidosis was demonstrated (Table I). On examination there were no significant neurologic signs. Urinalysis showed an aciduria, minimal proteinuria, and strong acetonuria. The excretion of urinary porphyrins and amino acids was normal. After treatment with an intravenous bicarbonate and electrolyte solution, recovery was rapid.

The child was again admitted in coma when he was 48 months old after a 2-day illness with sore throat and fever. In the day prior to admission, he vomited 15 times. A macular rash attributed to rubella was noted. Profound metabolic acidosis was again documented. Routine chemistries were all normal and plasma and urinary amino acids were within normal limits. The Rothera test for "acetone" in plasma and urine was strongly positive. He was again treated for metabolic acidosis.

Another admission with similar signs and symptoms occurred when the patient was 58 months of age. On this occasion, referral to our group led to the discovery of large amounts of an unusual substance ( $\alpha$-methyl $-\beta$ hydroxybutyric acid) in his urine. There were two subsequent admissions for mild acidosis precipitated by intercurrent febrile illness (Table I). In the interim the diagnosis of a disorder of isoleucine catabolism had caused us to recommend the use of a low protein diet $(2 \mathrm{~g} / \mathrm{kg} / 24 \mathrm{hr})$ as well as early management of incipient acidosis; the family was counselled concerning the nature of the illness. The child has been well since and physical and mental growth have remained normal. The parents are Dutch immigrants to Canada and are not consanguinously related. The propositus is the first to be affected within the pedigree to our knowledge.

\section{$M$ Pedigree}

The $M$ family came to Canada in 1971 , having emigrated from Chile. Patient M,VII,5 is the propositus. Her parents are related via a distant common ancestor.

The propositus was born in 1967 after a 34-week gestation, when delivery by Cesarian section was performed for "severe maternal toxemia." The infant weighed 2,400 $\mathrm{g}$ at birth and was apparently well until 1 year of age when she was admitted for 1 week to hospital with fever, hematemesis, and bloody diarrhea. She developed as a thin child "who liked neither milk or meat." The patient first came to attention at the Montreal Children's Hospital at 4 years of age because of vomiting and acetonuria. She was referred to our group at which time a substance was identified in the urine with the identical retention time and mass spec- 
trum of the "unknown" material excreted by the propositus of the B pedigree. She presented again with vomiting and acetonuria at 5 years of age when preventive management was carried out with dimenhydrinate and protein restriction; the crisis resolved in 24 hr. Physical and mental growth are normal.

A sister of the propositus (patient $M, V I I, 3$ ) was born in 1962 after a normal gestation. Delivery was by footling breech after elective induction of labor. She was apparently well until 12 monts of age when she was admitted to a hospital elsewhere with fever, vomiting, and hyperventilation. Coma, hematemesis, and bloody diarrhea ensued and a sweetish odor to the breath was noted. In spite of therapy, she remained unconscious, convulsed repeatedly, and died on the 3rd hospital day.

A brother of the propositus (patient $M, V I I, 4$ ) was born in 1963. His gestation was normal but, because of fetopelvic disproportion, delivery was performed under general anesthetic. He was breast fed until 4 months of age when he began refusing the nipple; bottle feedings were refused as well. Continued milk feedings were possible only after persistent offerings. At 12 months, he was admitted to a hospital with a short history of fever, hematemesis, and diarrhea. Within hours he had lapsed into a coma which endured 3 days. Convulsions were noted during this period. At 15 months, a similar episode occurred. At 36 months he was admitted to another hospital for similar reasons. After 4 days of coma, his condition slowly improved. Two months later he was readmitted for another episode. During this last admission, he suffered a cardiac arrest but was resuscitated. About six more acidotic episodes have occurred, all of less severity. At age 8 years, the family moved to Montreal when the child came to our attention, and the diagnosis was made upon discovery of $\alpha-\mathrm{M} \beta \mathrm{HB}$ and $\alpha$-MAA in his urine. Examination indicates that he has suffered psychomotor damage, probably as a sequel to the comatose episodes.

\section{Materials and Methods}

\section{Identification of Organic Acids}

Organic acids were extracted from urine by the method of Mamer et al. [9]. Silylated ether extracts $(1-2 \mu l)$ were analyzed on a gas chromatograph-mass spectrometer coupled unit [16]. Column conditions were: glass column ( 6 feet by 0.25 inch) packed with SE-30 3\% Ultraphase on Chromosorb W (HP), 80/100 mesh [17] flash heater at $275^{\circ}$, temperature programming $80-280^{\circ}$ at $4^{\circ} / \mathrm{min}$ with no delay, flow rate of helium $30 \mathrm{ml} / \mathrm{min}$. The separator operated at $280^{\circ}$ with an ion source temperature of $290^{\circ}$. Ionizing energy and current were $70 \mathrm{eV}$ and $60 \mu \AA$, respectively. Scan time was $4 \mathrm{sec}$ between $m / e 60$ and 600 . Acceleration potential was $3.5 \mathrm{kV}$. After identification of the unknown material, all subsequent investigation was carried out with a gas chromatograph [18] operated under identical conditions.

\section{Amino Acid Analysis}

Quantitative analysis of amino acids in plasma ( 1 volume) deproteinized with sulfosalicylic acid (3\% $\mathrm{w} / \mathrm{v}$ ) (5 volumes), was performed on an amino acid analyzer [19] modified for rapid analysis of branched chain amino acids according to the methods of Scriver et al. [15].

\section{Ketone Analysis}

Urinary and plasma ketones were analyzed as their 2,4-DNPH derivatives by a modification of the method of Menkes [11]. Aliquots of urine were saturated with $\mathrm{NaCl}$ and alkalinized with $\mathrm{I} \mathrm{N} \mathrm{NaOH}$ to $\mathrm{pH}$ 10.5. Urinary ketones were then extracted three times into an equal volume of ether. The ether extract was evaporated to approximately 0.25 of the original volume, after which it was treated with a solution containing 2,4-dinitrophenylhydrazine (2,4-DNPH). The resultant 2,4-DNPH derivatives of the ketones were developed on silica gel sheets [20] in a chloroformhexane $(\mathrm{l}: \mathrm{l})$ solvent. The ketones appeared as yellow spots which were also visible under ultraviolet light. The identity of butanone was confirmed in numerous urine samples by cochromatography with an authentic standard and by extraction and subsequent gas chromatography.

\section{Amino Acid Loading Studies}

Three studies were undertaken, one each with $\mathrm{L}$-valine, L-leucine, and L-isoleucine. The purity of each amino acid used in these studies was confirmed by chromatographic analysis; the leucine preparation contained no isoleucine contaminant. The amino acid was given 6 times, as a powder with meals, $75 \mathrm{mg} / \mathrm{kg}$ each dose. Each period of dietary loading lasted 2 days. Two days more were allowed between the loading periods so that excretion of organic acid derivatives could return to base line levels. All urine was collected in 8-hr periods throughout the loading studies, and 
analyzed for acetone and organic acids. The excretion of metabolites was compared with preload control samples.

\section{Partition Chromatography}

Partition chromatography of urine extracted into ether after acidification was carried out in an ascending direction during development in a mixture of butanol, acetic acid, and water $(12: 3: 5)$ on Whatman no. 1 chromatography paper. The chromatogram was stained with bromocresol green $(50 \mathrm{mg}$ in $100 \mathrm{ml}$ butanol and $l \mathrm{~N} \mathrm{NaOH}$ ) to rule out the presence of methylmalonic acid, a substance whose elution position in the gas-liquid chromatography (GC) system is close to that of $\alpha-\mathrm{M} \beta \mathrm{HB}$.

\section{Cullured Skin Fibroblast Studies}

Fibroblasts were cultured from skin biopsy explants and incubated at $37^{\circ}$ in Eagle's minimum essential medium, containing $12 \%$ fetal calf serum under an atmosphere of $5 \% \mathrm{CO}_{2}$ in air. Fibroblasts from normal patient cell lines were taken at comparable age in culture and from donors of similar age. Cultures were rinsed three times with cold saline and buffered with $5 \mathrm{ml}$ Tris buffer, pH 7.4, after which the monolayers were scraped with a rubber policeman and about 2 million cells (or $5 \mathrm{mg}$ protein) were placed in a Warburg flask. Incubation for $1 \mathrm{hr}$ was carried out in the presence of substrate ( $0.4 \mathrm{~mm}$ final concentration). L-
Isoleucine, L-leucine, and succinate were obtained from Mann Chemical Corporation [21]. Uniformly ${ }^{14} \mathrm{C}$ labeled L-isoleucine (sp act, $318 \mathrm{mCi} / \mathrm{mmol}$ ), L-leucine (sp act, $296 \mathrm{mCi} / \mathrm{mmol}$ ), and succinate (sp act, 108 $\mathrm{mCi} / \mathrm{mmol}$ ) were obtained from New England Nuclear Corporation [22]. The reaction was stopped and $\mathrm{CO}_{2}$ release initiated by acidification with $50 \mu \mathrm{H}_{2} \mathrm{SO}_{4}$ per flash tipped from the side arm. The $\mathrm{CO}_{2}$ was collected on filter paper soaked in $1 \mathrm{~m} \mathrm{KOH}$ for $1 \mathrm{hr}$ with gentle shaking; the efficiency of $\mathrm{CO}_{2}$ collection by this method is $59 \% \pm 4 \%$. The paper was dried and placed in 0.4 $\mathrm{ml}$ Hyamine, to which were added $10 \mathrm{ml}$ scintillation fluid, and counted in a scintillation counter [23]. Protein content of the cell preparations was determined by the method of Lowry et al. [8].

\section{Results}

\section{Identification of $\alpha$-Methyl- $\beta$-hydroxybutyric Acid}

Gas chromatographic analysis of urine collected from patients during episodes of acidosis revealed a large unusual peak (retention time of approximately $7.6 \mathrm{~min}$ in the gas chromatogram [16], Fig. $2 A$ ) with a mass spectrum suggestive of a bis-trimethylsilyl (TMS) derivative of an $\omega$-less-1-hydroxy, 5-carbon aliphatic acid (Fig. 2A). The identity of the unknown was suspected after elimination of other alternatives by spectrum comparisons and finally confirmed as $\alpha-\mathrm{M} \beta \mathrm{HB}$ by comparisons with that compound obtained by rational
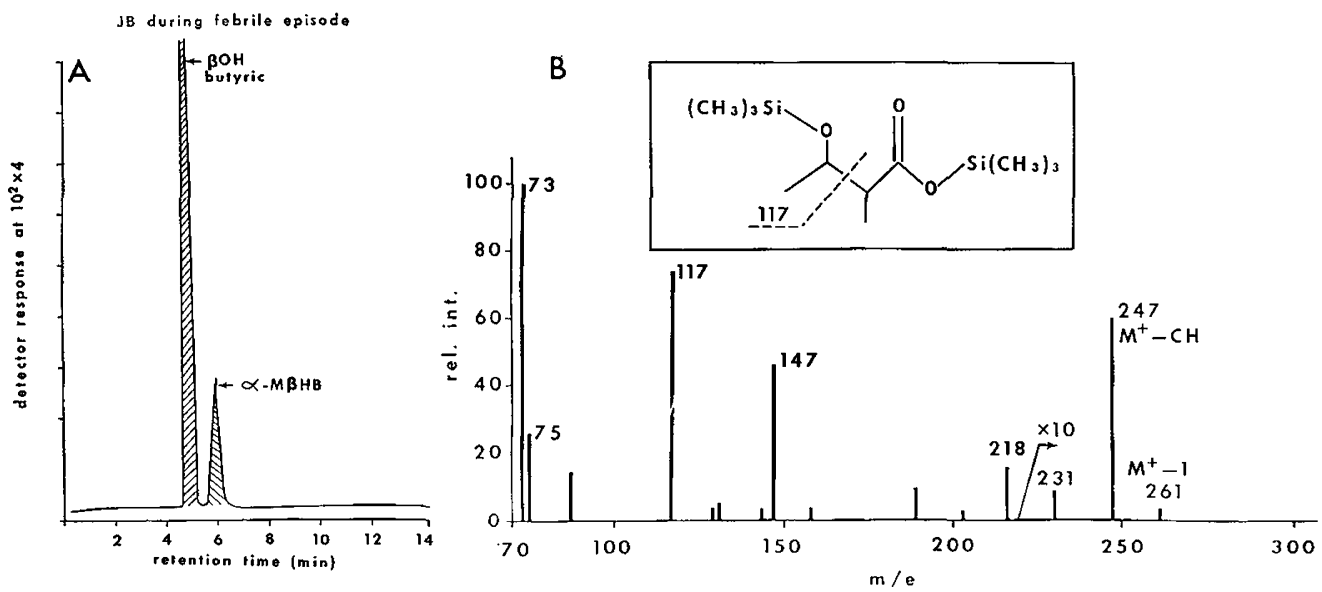

Fig. 2. A: Gas-liquid chromatogram of trimethylsilylated (TMS) derivatives in urine from proband $B, I I I, 2$ during a febrile episode. This chromatogram shows two significant peaks: $\beta$-hydroxybutyric acid ( $\beta O H$ butyric), which is a "normal ketone body", and $\alpha$-methyl- $\beta$-hydroxybutyric acid $(\alpha-M \beta H B)$, the abnormal metabolite. Note that the retention time as shown was obtained on the Hewlett-Packard instrument; this system of analysis is slightly different from that quoted in the text which was derived from the LKB-9000 gas chromatograph-mass spectrometer. $B$ : Mass spectrum of $\alpha$-methyl- $\beta$-hydroxybutyric acid, bis-trimethylsilylated (bis-TMS) derivative. Significant ions are $m / e$ 247, representing loss of a TMS methyl group by the molecular ion $(\mathrm{m} / \mathrm{e} 262), \mathrm{m} / \mathrm{e} 147$ assigned to a rearrangement ion characteristic of compounds incorporating two or more TMS groups, and $m / e 117$, indicative of hydroxyl substitution on the next-to-terminal carbon atom. 
synthesis (monomethylation of acetoacetic ester with methyliodide in ethanolic sodium ethoxide followed by sodium borohydride reduction and mild alkaline hydrolysis to the free acid). The unknown and the authentic $\alpha-\mathrm{M} \beta \mathrm{HB}$ as the TMS derivatives cochromatograph and have identical mass spectra. The $\alpha-\mathrm{M} \beta \mathrm{HB}$ is a metabolite in the catabolic pathway of isoleucine.

Urine collected between episodes of metabolic acidosis in all patients contained $\alpha-\mathrm{M} \beta \mathrm{HB}$ at all times and greatly elevated amounts during bouts of acidosis. (Relative peak size per milligram of urinary creatinine was used as a coefficient inasmuch as a pure standard of synthetic $\alpha-\mathrm{M} \beta \mathrm{HB}$ was not available for quantitation.) Plasma contained a very small but abnormal amount of $\alpha-\mathrm{M} \beta \mathrm{HB}$ during bouts of acidosis.

This acid has subsequently been found to occur in trace quantities in urine from normal healthy subjects.

\section{Identification of $\alpha$-Methylacetoacetic Acid}

Analysis of urine from $\mathrm{B}$ and $\mathrm{M}$ propositi yielded a second abnormal substance appearing as two peaks with retention times of 9 and $10.4 \mathrm{~min}$ on the GC system [16] (Fig. 3A). This compound was also analyzed by mass spectrometry and identified as $\alpha$-MAA (Fig. $3 B$ ). When synthesized (an intermediate in the $\alpha$ $\mathrm{M} \beta \mathrm{HB}$ synthesis), this compound cochromatographed and had spectra identical with the unknown. This compound was appearently present in smaller quantities than $\alpha-\mathrm{M} \beta \mathrm{HB}$ as judged by the relative peak sizes

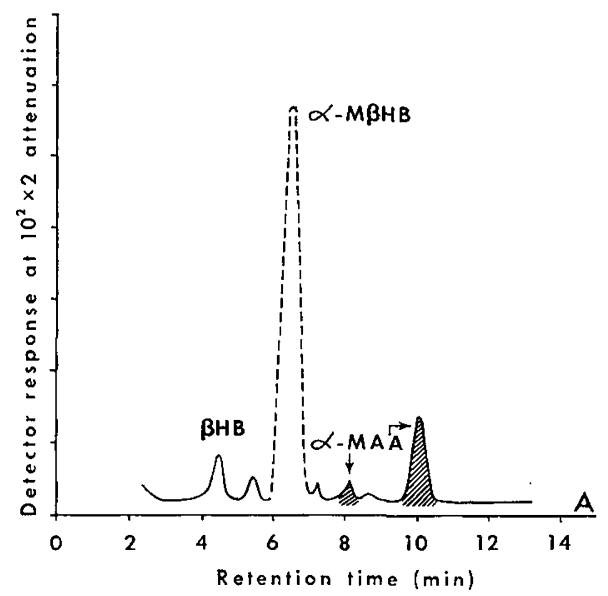

of the two substrates. Enol formation during silylation accounts for the two peaks of $\alpha$-MAA revealed by gas chromatography. Both peaks have identical mass spectra. This acid is rarely seen in normal urines in even trace quantities.

\section{Identification of N-Tiglylglycine}

The $M$ propositus excreted a third unusual substance in the urine, the retention time of which was I5.6 min on the LKB-9000 system. This metabolite had a mass spectrum identical with $N$-tiglylglycine (Fig. 4) and chromatographed in its place. This conjugated metabolite in the isoleucine pathway (Fig. 1) was excreted at all times in the $M$ probands but never in the $B$ propusitus. Its excretion in the $M$ probands is consistently augmented during acidotic interludes. This conjugate is not found in normal urines.

\section{Identification of Amino Acids and Other Organic Acids}

Blood and urine levels of all amino acids were consistently normal. Hyperglycinemia was never apparent even during ketotic acidosis.

Propionate levels in the urine and plasma of $\mathbf{B}$ and $M$ probands were within normal limits. There was no evidence for excessive accumulation of the keto acid of isoleucine $(\alpha$-keto- $\beta$-methyl valeric acid) or of its first oxidation product, $\alpha$-methylbutyric acid. $\beta$-Hydroxybutyric acid was identified in urine in large amounts only

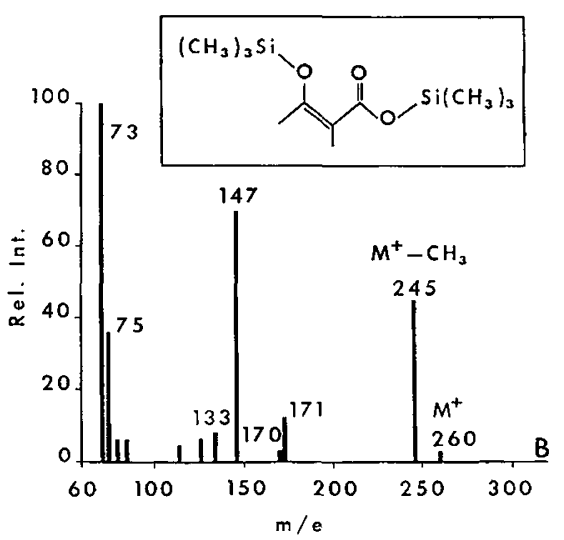

Fig. 3. A: Gas-liquid chromatogram of trimethylsilyI (TMS) derivatives in urine from proband $B, I I I, 2$ during a mild acidotic episode. This chromatogram shows the retention times of the two silylated isomers of $\alpha$-methylacetoacetate $(\alpha-M A A)$, and the relative position of $\alpha$-methyl- $\beta$-hydroxybutyric acid $(\alpha-M \beta H B)$. Minor technical differences again account for the discrepancy between times shown and those metioned in the text. $\beta H B: \beta$-Hydroxybutyric acid. $B$ : Mass spectrum of $\alpha$-methylacetoacetic acid. This $\beta$-keto acid characteristically yields a pair of bis-TMS derivatives on gas liquid chromatography, the first to elute being approximately $20 \%$ of the size of the second. Both peaks have identical mass spectra at $70 \mathrm{eV}$ ionizing energy and very similar spectra in the range $15-20 \mathrm{eV}$. This suggests that these two derivatives are closely related, perhaps via cis-trans isomerism about the enolic unsaturation. 


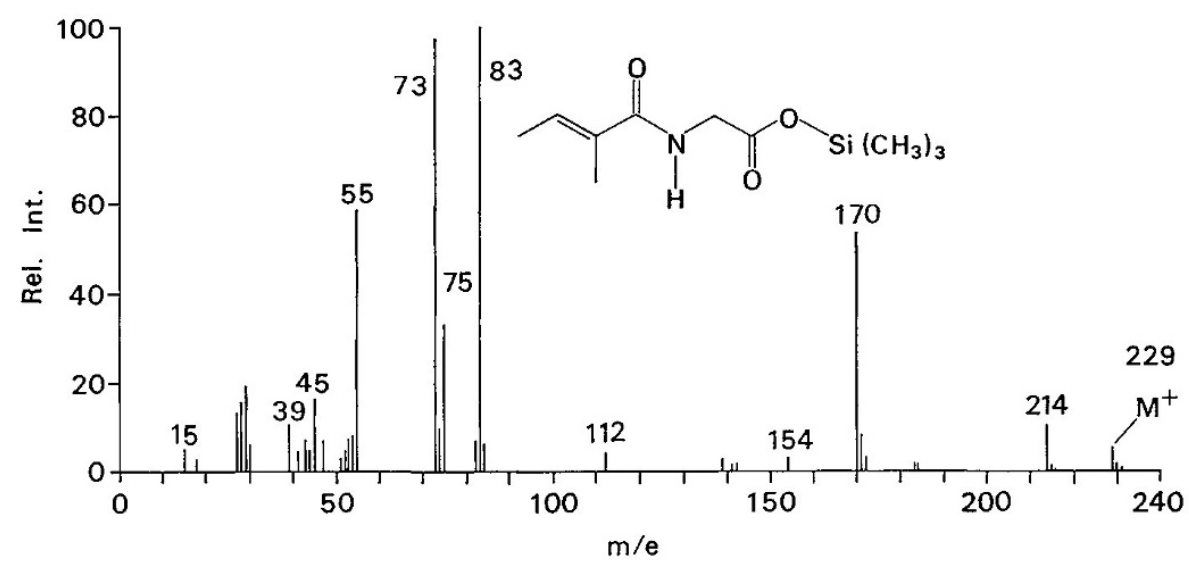

Fig. 4. Mass spectrum of the silylated derivative of $N$-tiglylglycine. This compound was present in the urine of patients in the $\mathrm{M}$ and $\mathrm{S}$ pedigrees. It is presumably formed by conjugation of free tiglic acid, a metabolite of isoleucine, with glycine. The base peak at $m / e$ represents the tiglyl fragment ion and a molecular ion at $m / e 229$ is evident. Rel. Int.: Relative intensity.

when the patients were acidotic. Hippuric acid was present in large amounts in the $M$ probands. Adipic acid was found in the urine in augmented amounts when acidosis prevailed in both propositi; the amount was diminished during periods of well-being.

\section{Urinary Ketones}

Urine obtained during periods of metabolic acidosis, and after loading with isoleucine contained butanone in significant quantities (Fig. 5). Acetone was also present, but pentanone and hexanone were not found at any time. Butanone produces a red-brown reaction with Acetest [24] tablets.

\section{Amino Acid Loading Studies}

The propositus of the B pedigree was studied by means of branched chain amino acid loading tests. The valine load did not augment the excretion of $\alpha-\mathrm{M} \beta \mathrm{HB}$ or $\alpha$-MAA. The leucine load caused a small increase in the urinary excretion of both compounds and isoleucine produced a dramatic increase in their excretion (Fig. 6). Blood gas analysis performed concurrently with the isoleucine load revealed modest metabolic acidosis when metabolite excretion was maximal on the 2 nd day of the loading test.

\section{Family Studies}

$B$ pedigree. Random daytime urine samples were collected from brothers and both parents of the propositus and analyzed for $\alpha-\mathrm{M} \beta \mathrm{HB}$. This metabolite was present in small but abnormal amounts in the urine of both parents and one sib. Addition of L-isoleucine to the diet $(75 \mathrm{mg} / \mathrm{kg}$ at each meal) for 2 days caused a significant increase in the excretion of $\alpha-\mathrm{M} \beta \mathrm{HB}$ in these subjects. Control subjects did not show this response. Neither the base-line levels of $\alpha-\mathrm{M} \beta \mathrm{HB}$ excretion nor the increase after loading in the parents was as great as in the propositus. The phenotypic pattern suggests that $\alpha-\mathrm{M} \beta \mathrm{HB}$ accumulation is an autosomal recessive trait. Excretion of $\alpha$-MAA was not abnormal in the presumed heterozygotes.

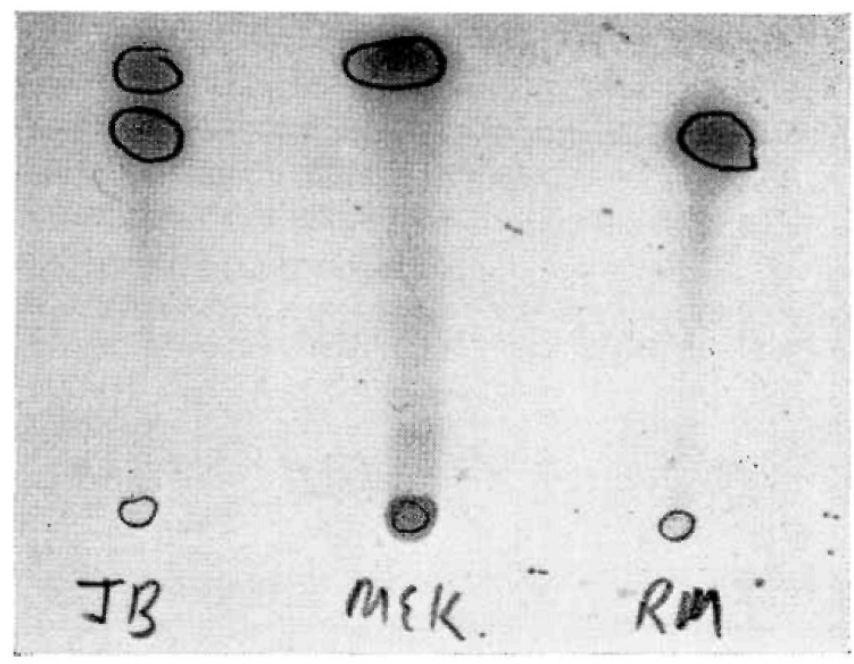

Fig. 5. Thin layer chromatogram on silica gel of urinary ketones as 2,4-DNPH derivatives. $J B$ is proband $B, I I I, 2, R M$ is a normal control subject. MEK is the 2,4-DNPH derivative of methyl ethyl ketone, used as the butanone standard. The unknown substance in the patient's urine also cochromatographed with MEK when the latter was added to the patient's urine. The butanone derivative on the silica gel sheet is yellow in visible light and Iavender in ultraviolet light. The patient's unknown spot exhibited similar characteristics. 


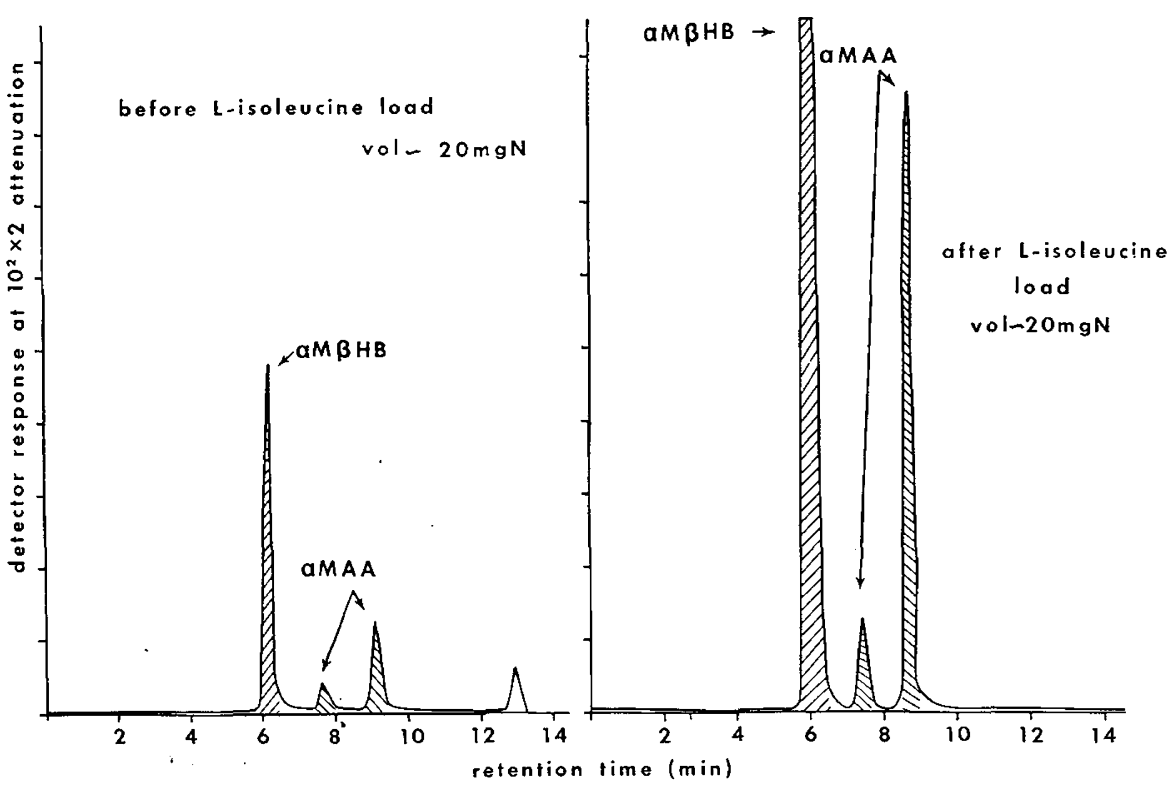

Fig. 6. Response to isoleucine loading. The gas-liquid chromatogram on the left was derived from a urine specimen prior to the "chronic" isoleucine load (see text). On the right is a chromatogram derived from a urine specimen taken in the immediate $24 \mathrm{hr}$ after the last dose. Note the dramatic increase 'in peak size of the abnormal metabolites associated with isoleucine feeding. $\alpha-M B H B: \alpha$-Methyl- $\beta$-hydroxy butyric acid; $\alpha-M A A: \alpha$-methylacetoacetic acid.

$M$ pedigree. A brother of the propositus excretes large amounts of $N$-tiglylglycine, $\alpha-\mathrm{M} \beta \mathrm{HB}$, and $\alpha$-MAA as well as butanone during acidosis; one other sib and both parents excrete lesser but still abnormal amounts of $\alpha-\mathrm{M} \beta \mathrm{HB}$ only in their urine. The findings suggest that the former individual is probably homozygous and the latter heterozygous for the trait.

The apparent genotypes and their inheritance in the two pedigrees are indicated in Fig. 7. Consanguinity in the $M$ pedigree increases the likelihood that a rare mutant allele has been inherited in autosomal recessive fashion in this family. The possibility that the mutant allele is different in the $B$ and $M$ families has not been excluded.

\section{Fibroblast Studies}

Cultured skin fibroblasts from the propositi of both pedigrees grew well and showed no morphologic abnormalities in culture. The metabolic potential of $\mathrm{B}$ cells was studied in preliminary fashion. The ability to oxidize $\mathrm{L}$-isoleucine- $\mathrm{U}-{ }^{14} \mathrm{C}$ to ${ }^{14} \mathrm{CO}_{2}$ was impaired in the mutant cells in comparison with control cells grown through a similar number of generations to the same density and harvested at the same time after subculture (Table II). There was no defect in the oxidation of succinate. These observations, coupled with the metabolic studies in vivo, presumably place the defect of isoleucine oxidation in the pathway at some point beyond decarboxylation but prior to the oxidation of propionyl-CoA. The effect of temperature upon isoleucine catabolism was determined in vitro because of the frequent clinical correlation between fever and acidotic episodes. Incubations at $40^{\circ}$ and at $42^{\circ}$ did not depress isoleucine oxidation below that observed at $37^{\circ}$ in the cultured fibroblasts.

\section{Discussion}

Suspicion that our patients had organic aciduria started with their histories of recurrent acidotic episodes without obvious cause. The clinical evidence suggests that this "new" metabolic aberration need not, of its own accord, be harmful, but that the severe (uncompensated) acidotic episodes which the patient is likely to experience can be severe enough to cause mental retardation or even death. Patients $B, I I I, 2$ and $M, V I I, 5$ have developed normally although they appear to be homozygous for the mutant allele. On the other hand, patient $M, V I I, 4$ is retarded mentally after a series of severe acidotic episodes, one of which was complicated by cardiac arrest. Patient $M, V I I, 3$ died during an acidotic episode, presumably a manifestation of the same inborn error of metabolism inherited by her sibs.

The clinical onset of disease in our patients was late in the 1st year of life or thereafter. All the children 


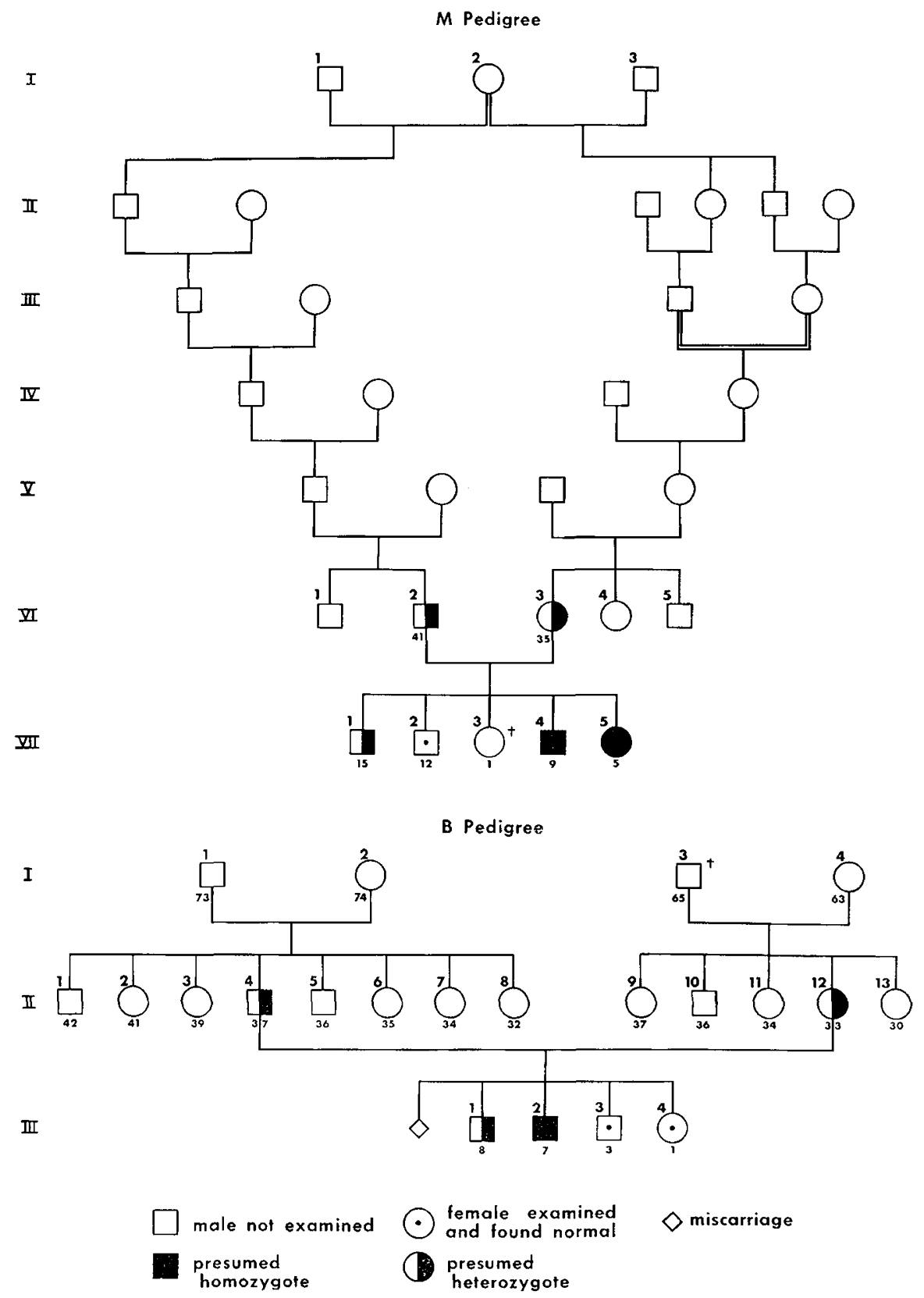

Fig. 7. Relevant pedigrees for the $\mathrm{M}$ and $\mathrm{B}$ propositi (patients $M, V I I, 4$ and $B, I I I, 2$, respectively). Assignment of the presumed genotype is based on the biochemical phenotype, determined by gas-liquid chromatography analysis of urine samples. Presumed homozygotes excrete large amounts of $\alpha$-methyl- $\beta$-hydroxybutyric acid $(\alpha-M \beta M B)$ and $\alpha$-methylacetoacetic acid (and also $N$-tiglylglycine in the $M$ pedigree); presumed heterozygotes excrete a small excess of $\alpha-\mathrm{M} \beta \mathrm{MB}$ only. L-Isoleucine feeding augments excretion of the unusual metabolities.

were breast fed and, because the isoleucine content of human milk is considerably lower than that of cow milk [3], the patients may have been protected from an earlier precipitation of metabolic acidosis. Intolerance to high protein intake is apparent in all of the patients and each has avoided severe episodes of acidosis, after the diagnosis was established, by restricting dietary protein intake.

The $\alpha-\mathrm{M} \beta \mathrm{HB}$ was detected at all times in the urine in affected probands, the amount being increased during periods of acidosis. However, precise quantitation of this and the other unusual metabolites has been 
Table II. Metabolism of ${ }^{14} \mathrm{C}$-labeled substrate in cultured skin fibroblasts of $\mathrm{B}$ propositus (percentage of values for control subjects) ${ }^{x}$

\begin{tabular}{lcccc}
\hline & $\begin{array}{c}\text { U-14 C-I-Ile } \\
(\mathrm{A})\end{array}$ & $\begin{array}{c}\text { U-14 C-X-Ile } \\
(\mathrm{B})\end{array}$ & $\begin{array}{c}\text { U-14 C-I- } \\
\text { Leu }\end{array}$ & $\begin{array}{c}1,4-{ }^{14} \mathrm{C}- \\
\text { Succinate }\end{array}$ \\
\hline $\begin{array}{l}\text { Distribution } \\
{ }^{14} \mathrm{C}\end{array}$ & & & & \\
$\quad$ In $\mathrm{CO}_{2}$ & 44 & 46 & 113 & 106 \\
$\quad$ In protein & & 94 & 94 & \\
\end{tabular}

${ }^{1}$ Experiments with mutant cultured skin fibroblasts from patient $B, I I I, 2$ and fibroblasts from a control subject of similar age were carried out as described under Materials and Methods. The data represent the number of counts from ${ }^{14} \mathrm{CO}_{2}$ released by mutant cells expressed as a percentage of the corresponding counts released by control cells. Both cell lines were in loggrowth phase and of similar culture age. Incorporation of labeled amino acids into the trichloroacetic acid-precipitable fraction (protein) was measured at the end of the experiment. The metabolism of leucine and succinate to $\mathrm{CO}_{2}$ is apparently normal in our patients' fibroblasts.

hampered by the lack of pure reference standards. One of us $(O M)$ has now accomplished a method of synthesis to yield pure $\alpha-\mathrm{M} \beta \mathrm{HB}$, which allows some preliminary quantitation. During acidosis the urinary excretion of $\alpha-\mathrm{M} \beta \mathrm{HB}$ in the presumed homozygotes exceeds $1 \mathrm{~g} / \mathrm{g}$ creatinine, whereas, at other times, it is in the range of $250-1000 \mathrm{mg} / \mathrm{g}$ creatinine.

The precipitating stimuli for acidosis and coma are unknown. Each episode was associated with febrile illness, at which time protein catabolism could release large amounts of endogenous isoleucine from tissue stores. Our in vitro studies with cultured fibroblasts do not reveal evidence for a temperature-sensitive abnormality of isoleucine metabolism. It is possible that, in the presence of impaired isoleucine catabolism, further input of the amino acid during febrile catabolic episodes causes accumulation of metabolites which in turn further inhibit isoleucine oxidation. Hillman et al. [6] recently described a disorder of isoleucine metabolism which resembles the present condition except for an associated hyperglycinemia. They suggested that tiglic acid accumulation was responsible for the hyperglycinemia in his patient. There was no hyperglycinemia in our patients on any occasion; whether tiglic acid inhibits isoleucine oxidation in our patients' tissues has not yet been confirmed.

The metabolite which appears to be present in greatest amounts in the urine is $\alpha-\mathrm{M} \beta \mathrm{HB}$, whereas $\alpha$ MAA is apparently present in lesser but still abnormal amounts. $N$-Tiglylglycine, when present, yields the smallest abnormal peak. These estimates are based on relative peak size and they assume that equimolar amounts of the three metabolites would yield approximately the same peak size on the gas chromatogram, a reasonable assumption because of their similar chemical structure. Contrary to the evidence suggested by relative peak size, the metabolic data favor a block at the thiolase step of isoleucine oxidation, that is at the step where $\alpha$-MAA is converted to propionyl-CoA and acetyl-CoA. The preferential accumulation of a metabolite which is a precursor of the substrate for the presumably deficient enzyme requires explanation. The situation may be analogous to the metabolic interrelations of "ketone bodies," ( $\beta$-OH-butyrate and acetoacetate) where the equilibrium between them in liver favors formation of the butyrate derivative [7]. Alternatively, an otherwise minor pathway of $\alpha$-MAA metabolism may exert its influence on accumulated $\alpha$-MAA in the mutant state and remove it; the excess of butanone in our patients indicates at least one route by which this can occur; on the other hand, $\alpha-\mathrm{M} \beta \mathrm{HB}$ may be excreted preferentially over $\alpha$-MAA. Since the enzymes which interconvert tiglic acid, $\alpha-\mathrm{M} \beta \mathrm{HB}$, and $\alpha$-MAA are all reversible (Fig. 1), accumulation of tiglic acid presumably reflects a new steady state between the three steps of isoleucine oxidation in our patients. We assume that formation of $\mathrm{N}$-tiglylglycine is a detoxifying mechanism to rid the organism of this highly toxic organic acid. It is interesting to note that $N$-tiglylglycine is also present in abnormal amounts in $\beta$-methylcrotonylglycinuria of the biotin-responsive type $[4,5]$, and the nonconjugated tiglate derivative is also present in abnormal amounts in some patients with propionicacidemia [13]. The mechanism (or mechanisms) for accumulation of this potent metabolite in such diverse metabolic disorders encourages us to believe that several different metabolites may inhibit tiglic acid oxidation.

Although a catabolic pathway for isoleucine has been described and generally accepted (Fig. 1), the authenticity of the final steps involving the conversion of tiglyl-CoA through to acetyl-CoA and propionyl-CoA have not been confirmed in human tissues. It was presumed by Robinson et al. [14] that the conversion steps proceeded as shown because of the close parallel with $\beta$-oxidation of fatty acids and because of their own experimental evidence. The findings in our patients suggest that the pathway as it is known also carries out the final stages of isoleucine oxidation in man, but confirmation must rest on specific studies in vitro. 
If the defective enzyme is the thiolase in the isoleucine pathway we cannot say yet whether it is a primary defect related to a mutation in the "thiolase" gene or a secondary defect reflecting inhibition of unspecified nature. The former hypothesis introduces the corollary that the $\alpha$-MAA thiolase must be specific, both in substrate preference and genetic control, because other thiolase (EC 2.3.1.16)-catalyzed metabolism [2] (e.g., acetoacetate metabolism) is normal. The organ or tissue site of the defect in our patients is also unknown. Brached chain amino acid oxidation is an important function of skeletal muscle [12], but whether the present trait is a disorder of muscle and liver or of both tissues is unknown.

The pedigree studies indicate that the trait which affects isoleucine catabolism is inherited, apparently in autosomal recessive fashion. The probands are presumably homozygous, but presumed heterozygotes, who are otherwise healthy, show some biochemical features of the trait. Whether the mutant allele is the same in B and $M$ pedigrees is unknown and whether the trait (or traits) described here is identical with that reported in abstract by Hillman et al. [6] is also not known. We have had the occasion to examine urine from yet another propositus, this time in a Greek family [25]. The GC analysis and clinical features suggest that the $\mathrm{S}$ propositus resembles our $\mathrm{M}$ probands more than the $\mathrm{B}$ propositus. Family studies have not yet been carried out in the $S$ pedigree. We presume that the $M$ probands inherited a pair of rare but similar mutant alleles because of the consanguinity in their pedigree. However, the B pedigree, which to the best of our knowledge is not consanguinous, could contain a dissimilar pair of mutant alleles at the same genetic locus, which when paired produce a "genetic compound" [10] with an apparently homozygous phenotype. Specific evaluation of the presumed enzyme defect in vitro and performance of the appropriate kinetic studies may discern the extent of genetic heterogeneity in this trait.

\section{Summary}

A "new" disorder of isoleucine catabolism is described. The block in oxidation apparently precedes the stage of propionyl-CoA formation and is beyond oxidative decarboxylation of $\alpha$-keto- $\beta$-methylvalerate. The coexistent accumulation and excretion in urine of large amounts of $\alpha$-methylacetoacetate, $\alpha$-methyl $-\beta$-hydroxybutyrate, $N$-tiglylglycine, and butanone suggests that the block occurs at the "thiolase" step converting $\alpha$ -
methylacetoacetyl-CoA to acetyl-CoA and propionylCoA. The accumulation of three different metabolites in the presumed isoleucine catabolic pathway may reflect altered equilibrium of reversible interconversion reactions.

The clinical manifestations of the condition are intermittent vomiting, metabolic acidosis, obtunded alertness, and coma. The clinical course is ameliorated by a reduction of protein intake in the diet to about 2 $\mathrm{g} / \mathrm{kg} / 24 \mathrm{hr}$ and aggressive care of infections. L-Isoleucine feeding specifically aggravates the biochemical and metabolic perturbations.

Examination of two pedigrees suggests that the condition is inherited in autosomal recessive fashion. Presumed obligate heterozygotes excrete $\alpha$-methyl- $\beta$-hydroxybutyrate in modest excess in the urine. A third and fourth propositus are known to us who resemble in some aspects, the two propositi reported in detail here. The possibility of genetic heterogeneity among probands in different pedigrees is quite likely.

\section{References and Notes}

1. Daum, R. S., Lamm, P. H., Mamer, O. A., and Scriver, C. R.: A "new" disorder of isoleucine matabolism. Lancet, $i$, 1289 (1971).

2. Dierks-Ventling, C., And Cone, A. L.: Acetyl-coenzyme A thiolase in brain liver and kidney during maturation of the rat. Science, 172: 380 (1971).

3. Fomon, S. J.: Infant Nutrition. (W. B. Saunders Company, Philadelphia, 1967).

4. Gompertz, D., and Draffan, G. H.: The identification of tiglylglycine in the urine of a child with $\beta$-methylcrotonyl glycinuria. Clin. Chim. Acta, 37: 405 (1972).

5. Gompertz, D., Draffan, G. H., Watts, J. L., and Hull, D.: Biotin responsive $\beta$-methylcrotonylglycinuria. Lancet, $i i: 22$ (1971).

6. Hillman, R. E., Feign, R. D., Tenenbaum, S. M., and Keating, J. P.: Defective isolucine metabolism as a cause of the "ketotic hyperglycinemia" syndrome (Abstract). Pediat. Res., 6: 394 (1972).

7. Lehninger, A. L.: Biochemistry. (Worth Publishers, Inc., New York, 1970).

8. Lowry, O. H., Rosebrough, N. J., Farr, A. L., and Randall, R. J.: Protein measurements with the folin phenol reagent. J. Biol. Chem., 193: 265 (1951).

9. Mamer, O. A., Crawhall, J. C., and TJon, S. S.: The identification of urinary acids by coupled gas chromatographymass spectrometry. Clin. Chim. Acta, 32: 171 (1971).

10. McKusick, V. A., Howell, R. R., Hussels, I. E., Neufeld, E. F., ANd Stevenson, R. E.: Allelism non-allelism and genetic compounds among the mucopolysaccharidoses. Lancet, i: 993 (1972).

11. Menkes, J. H.: Idiopathic hyperglycinemia: isolation and identification of three previously undescribed urinary ketones. J. Pediat., 69: 413 (1966). 
12. MrLler, L. L.: The role of the liver and the non-hepatic tissues in the regulation of free amino acid levels in the blood. In: J. T. Holden: Amino Acid Pools, p. 708. (American Elsevier Publishing Company, New York, 1962).

13. Nyhan, W. L., Ando, 'T., Rasmussen, K., Nadlington, W., Kulrox, A. W., Cortom D., and Hull, D.: Tiglicaciduria in propionic acidaemia. Biochem. J., 126: 1035 (1972).

14. Robinson, W. G., BachHawat, B. K., and Coon, M. J.: Tiglyl-coenzyme A and $\alpha$-methylacetoacetyl coenzyme A, intermediates in the enzymatic degradation of isoleucine. J. Biol. Chem., 218: 391 (1956).

15. Scriver, C. R., Davies, E., and Lamm, P.: Accelcrated selective short column chromatography of neutral acidic amino acids on a Beckman-Spinco Analyzer, modified for simultaneous analysis of the samples. Clin. Biochem., 1: 179 (1968).

16. LKB 9000, LKB Instruments, Inc., Rockville, Md.

17. Pierce Chemical Company, Rockford, Ill.

18. No. 402, Hewlett-Packard, Avondale, Penna.

19. Beckman-Spinco, Palo Alto, Calif.

20. Eastman Kodak Company, Rochester, N. Y.

21. Mann Chemical Corporation, Orangeburg, N.Y.

22. New England Nuclear Corporation, Boston, Mass.

23. Unilux, Nuclear Chicago Corporation, Des Plaines, Ill.

24. Ames Company, Division of Miles Labs., Inc., Elkhart, Ind.

25. S pedigree, identified by Dr. Catherine Dacon-Vouletakis, Department of Pediatrics, Aglua-Sophia Children's Hospital, Athens, Greece.

26. The nature of these studies was explained to the parents of the $M$ and $B$ probands and the studies were carried out with the informed consent of the responsible individuals.
27. We are grateful to Carol Clow, Ines Koc, and Terry Reade for their assistance in the investigation of the patients and their families. Dr. Harvey Guyda and Dr. Michael Klein kindly referred the propositi in the $M$ and $B$ pedigrees, respectively. Dr. Hy Goldman generously grew the skin fibroblast cultures and performed the skin biopsies for us. Dr. David Harpp of the Department of Chemistry and Dr. John C. Crawhall (Department of Medicine) offered helpful discussion of various aspects of the problem. Mrs. Huguette Ishmael provided valuable editorial assistance.

28. Presented in part at the Annual Meeting of the Society for Pediatric Research, Atlantic City, May 1971. Daum, R., Delvin, E., Goldman, H., Lamm, P., Mamer, O. and Scriver, C.: A new inherited defect of isoleucine catabolism (Abstract). Pediat. Res., 5: 392 (1971).

29. Dr. R. Daum performed the studies in partial fulfillment of the requixements for an M.Sc. degree in the Department of Biology (Human Genetics Sector).

30. Publication no. 348 from the McGill University-Montreal Children's Hospital Research Institute.

31. This work was supported by grants from the Medical Research Council of Canada (no. MT-1085), The National Genetics Foundation (New York), and the Quebec Network of Genetic Medicine.

32. Requests for reprints should be addressed to: Charles $R$. SCRIVER, M.D. deBelle Laboratory for Biochemical Genetics. McGill University-Montreal Children's Hospital Research Institute, 2300 Tupper Street, Montreal 108, Quebec, Canada. 33. Accepted for publication November 27, 1972. 